\title{
CHARACTERIZATION OF CLASSICAL TYPE ORTHOGONAL POLYNOMIALS
}

\author{
K. H. KWON, L. L. LITTLEJOHN, J. K. LEE, AND B. H. YOO
}

(Communicated by Hal L. Smith)

\begin{abstract}
We characterize the classical type orthogonal polynomials $\left\{P_{n}(x)\right\}_{0}^{\infty}$ satisfying a fourth-order differential equation of type

$$
\sum_{i=0}^{4} \ell_{i}(x) y^{(i)}(x)=\lambda_{n} y(x)
$$
\end{abstract}

where $\ell_{i}(x)$ are polynomials of degree $\leq i$ and $\lambda_{n}$ is a constant. They are only the orthogonal polynomials satisfying an orthogonality of the form

$$
\left\langle\tau_{2}, P_{m}^{\prime \prime} P_{n}^{\prime \prime}\right\rangle+\left\langle\tau_{1}, P_{m}^{\prime} P_{n}^{\prime}\right\rangle+\left\langle\tau_{0}, P_{m} P_{n}\right\rangle=0 \quad \text { for } m \neq n
$$

where $\tau_{0}, \tau_{1}$, and $\tau_{2}$ are moment functionals.

\section{INTRODUCTION}

In 1929, Bochner [1] classified all orthogonal polynomial solutions to a second-order Sturm-Liouville differential equation of the form

$$
\ell_{2}(x) y^{\prime \prime}(x)+\ell_{1}(x) y^{\prime}(x)+\ell_{0}(x) y(x)=\lambda y(x) .
$$

They are, up to a complex linear change of variable, the classical orthogonal polynomials of Jacobi, Bessel, Laguerre, and Hermite.

Bochner's result naturally leads to a question of classifying all orthogonal polynomial solutions to higher-order differential equations of the form

$$
\sum_{i=0}^{N} \ell_{i}(x) y^{(i)}(x)=\lambda y(x) .
$$

In order for equation (1.2) to have polynomial solutions of degree $0,1, \ldots, N$, it must have the form

$$
\sum_{i=0}^{N} \sum_{j=0}^{i} \ell_{i j} x^{j} y^{(i)}(x)=\lambda_{n} y(x)
$$

Received by the editors May 15, 1992.

1991 Mathematics Subject Classification. Primary 33C45.

The first author was supported by the Korea Science and Engineering Foundation (Grant No. 90-08-00-02) and the Global Analysis Research Center during the preparation of this work. 
where

$$
\lambda_{n}=\ell_{00}+n \ell_{11}+n(n-1) \ell_{22}+\cdots+n(n-1) \cdots(n-N+1) \ell_{N N} .
$$

In 1938, H. L. Krall [6] found a necessary and sufficient condition for the differential equation (1.3) to have orthogonal polynomial solutions.

Theorem (H. L. Krall [6]). Let $\left\{P_{n}(x)\right\}_{0}^{\infty}$ be an orthogonal polynomial set. Then $P_{n}(x)$ satisfies the differential equation (1.3) for each $n=0,1,2, \ldots$ if and only if the moments $\left\{\sigma_{n}\right\}_{0}^{\infty}$ of $\left\{P_{n}(x)\right\}_{0}^{\infty}$ satisfy

$$
S_{k}(m)=\sum_{i=2 k+1}^{N} \sum_{j=0}^{i}\left(\begin{array}{c}
i-k-1 \\
k
\end{array}\right) P(m-2 k-1, i-2 k-1) \ell_{i, i-j} \sigma_{m-j}=0,
$$

$1 \leq 2 k+1 \leq N, m=2 k+1,2 k+2, \ldots$, where $P(n, k)=n(n-1) \cdots$ $(n-k+1)$. Furthermore, $N$ is necessarily even.

When $N=2 r, r \geq 1$, we call the $r$ equations in (1.5) the moment equations for $\left\{P_{n}(x)\right\}_{0}^{\infty}$. Further, in 1940, H. L. Krall [7] classified all fourth-order differential equations of the form (1.3) having orthogonal polynomial solutions. Up to a linear change of variable there are seven such equations, among which four are the iterations of the second-order differential equations satisfied by the four classical orthogonal polynomials and the other three have nonclassical orthogonal polynomial solutions. These polynomials were studied in detail by A. M. Krall [3] who named them the Jacobi type, Legendre type, and Laguerre type polynomials.

On the other hand, the four classical orthogonal polynomials are characterized as the only orthogonal polynomials whose derivatives also form orthogonal polynomials. It was first proved by Hahn [2] (see also H. L. Krall [5, 8] and Webster [13]).

In this work, we extend Hahn's theorem to include also the three classical type orthogonal polynomials satisfying fourth-order differential equations. This is the complete answer to the question in [12] (also raised by Professor W. N. Everitt at the 7th Symposium on Orthogonal Polynomials and Applications, Granada, Spain, 1991).

\section{MAIN THEOREM}

In this work, all polynomials are assumed to be real polynomials in one variable. We use $\operatorname{deg} \phi$ for the degree of a polynomial $\phi(x)$ and take $\operatorname{deg} 0$ to be -1 . We call any linear functional on the space of polynomials a moment functional. We denote the action of a moment functional $\sigma$ on a polynomial $\phi(x)$ by $\langle\sigma, \phi\rangle$ and call $\left\{\sigma_{n}\right\}_{0}^{\infty}$, where $\sigma_{n}:=\left\langle\sigma, x^{n}\right\rangle$, the moments of $\sigma$. For a moment functional $\sigma$ and a polynomial $\phi(x)$, we define the derivative $\sigma^{\prime}$ of $\sigma$ to be the moment functional defined by

$$
\left\langle\sigma^{\prime}, \psi(x)\right\rangle=-\left\langle\sigma, \psi^{\prime}(x)\right\rangle
$$

and the moment functional $\phi \sigma$ through the formula

$$
\langle\phi \sigma, \psi(x)\rangle=\langle\sigma, \phi(x) \psi(x)\rangle
$$

where $\psi(x)$ is a polynomial. Then it is easy to see that

$$
(\phi \sigma)^{\prime}=\phi^{\prime} \sigma+\phi \sigma^{\prime} \text {. }
$$


Two moment functionals $\sigma$ and $\tau$ are said to be equal and written $\sigma=\tau$ if $\langle\sigma, \phi\rangle=\langle\tau, \phi\rangle$ for any polynomial $\phi(x)$. Clearly, $\sigma=\tau$ if and only if they have the same moments. For example, if we define a moment functional $\sigma$ by

$$
\langle\sigma, \phi\rangle=\int_{0}^{\infty} \phi(x) \exp \left(-x^{1 / 4}\right) \sin x^{1 / 4} d x
$$

then $\sigma=0$; indeed, this result goes back to Stieltjes (see Widder [14, p. 126]).

Definition 2.1. A sequence of polynomials $\left\{P_{n}(x)\right\}_{0}^{\infty}$ is called an orthogonal polynomial set (OPS) if

(i) $\left\{P_{n}(x)\right\}_{0}^{\infty}$ is a polynomial set in the sense that $\operatorname{deg} P_{n}=n, n=0,1$, $2, \ldots$, and

(ii) there is a moment functional $\sigma$ such that

$$
\left\langle\sigma, P_{m} P_{n}\right\rangle=K_{n} \delta_{m n}, \quad m, n=0,1,2, \ldots,
$$

where $K_{n}$ are nonzero constants. Then we call $\sigma$ an orthogonalizing functional for the OPS $\left\{P_{n}(x)\right\}_{0}^{\infty}$.

Now, we are ready to state our main theorem.

Theorem 2.1. Let $\left\{P_{n}(x)\right\}_{0}^{\infty}$ be an OPS. Then, for each $n=0,1,2, \ldots, P_{n}(x)$ satisfies the fourth-order differential equation of the form

$$
L_{4}(y)=\sum_{i=0}^{4} \ell_{i}(x) y^{(i)}(x)=\sum_{i=0}^{4}\left(\sum_{j=0}^{i} \ell_{i j} x^{j}\right) y^{(i)}(x)=\lambda_{n} y(x)
$$

where $\ell_{4}(x) \not \equiv 0$ and $\lambda_{n}=\ell_{00}+n \ell_{11}+n(n-1) \ell_{22}+n(n-1)(n-2) \ell_{33}+$ $n(n-1)(n-2)(n-3) \ell_{44}$ if and only if there are moment functionals $\tau_{2} \not \equiv 0$, $\tau_{1}$, and $\tau_{0}$ such that

$$
\left\langle\tau_{2}, P_{m}^{\prime \prime} P_{n}^{\prime \prime}\right\rangle+\left\langle\tau_{1}, P_{m}^{\prime} P_{n}^{\prime}\right\rangle+\left\langle\tau_{0}, P_{m} P_{n}\right\rangle=M_{n} \delta_{m n}, \quad m, n=0,1,2, \ldots,
$$

where $M_{n}$ are constants.

We need the following lemma, which is essentially a restatement of $\mathbf{H}$. L. Krall's theorem in $\S 1$ for $N=4$ expressed in terms of an orthogonalizing functional instead of moments of an OPS.

Lemma 2.2. Let $\left\{P_{n}(x)\right\}_{0}^{\infty}$ be an OPS satisfying the differential equation (2.5) for each $n=0,1,2, \ldots$. Then a nonzero moment functional $\sigma$ is an orthogonalizing functional for $\left\{P_{n}(x)\right\}_{0}^{\infty}$ if and only if $\sigma$ satisfies

$$
2\left(\ell_{4} \sigma\right)^{\prime}-\ell_{3} \sigma=0
$$

and

$$
\left(\ell_{4} \sigma\right)^{(3)}-\left(\ell_{3} \sigma\right)^{\prime \prime}+\left(\ell_{2} \sigma\right)^{\prime}-\ell_{1} \sigma=0
$$

where 0 in the right-hand sides mean zero moment functionals.

The two equations (2.7) and (2.8) when they are understood as differential equations for a function are exactly the symmetry equations of which any nontrivial classical solution is a symmetry factor for the differential expression $L_{4}$ in (2.5) (see Littlejohn [10, 11] and A. M. Krall and Littlejohn [4]). They are used to yield the distributional weights by Littlejohn [11] for the classical type orthogonal polynomials (see also [9, Theorem 2.3]).

As an immediate consequence of Lemma 2.2, we have 
Corollary 2.3. Let $\left\{P_{n}(x)\right\}_{0}^{\infty}$ be an OPS relative to a moment functional $\sigma$ satisfying the differential equation (2.5) for each $n=0,1,2, \ldots$. Then for any polynomial $\phi(x)$ we have

$$
\left(L_{4} \phi\right) \sigma=\left[\phi^{\prime \prime} \ell_{4} \sigma\right]^{\prime \prime}-\left[\phi^{\prime}\left(\frac{1}{2}\left(\ell_{3} \sigma\right)^{\prime}-\ell_{2} \sigma\right)\right]^{\prime}+\phi \ell_{0} \sigma .
$$

Proof. Using (2.3), (2.7), and (2.8), it comes easily that

$$
\begin{aligned}
\left(L_{4} \phi\right) \sigma & =\phi^{(4)} \ell_{4} \sigma+\phi^{(3)} \ell_{3} \sigma+\phi^{\prime \prime} \ell_{2} \sigma+\phi^{\prime} \ell_{1} \sigma+\phi \ell_{0} \sigma \\
& =\left[\phi^{\prime \prime} \ell_{4} \sigma\right]^{\prime \prime}-\phi^{\prime \prime}\left(\ell_{4} \sigma\right)^{\prime \prime}+\left[\phi^{\prime} \ell_{2} \sigma\right]^{\prime}-\phi^{\prime}\left(\ell_{2} \sigma\right)^{\prime}+\phi^{\prime} \ell_{1} \sigma+\phi \ell_{0} \sigma \\
& =\left[\phi^{\prime \prime} \ell_{4} \sigma\right]^{\prime \prime}-\left[\phi^{\prime}\left(\frac{1}{2}\left(\ell_{3} \sigma\right)^{\prime}-\ell_{2} \sigma\right)\right]^{\prime}+\phi \ell_{0} \sigma .
\end{aligned}
$$

For later use, we note that (2.9) comes only from (2.7) and (2.8).

Lemma 2.4. Let $\left\{P_{n}(x)\right\}_{0}^{\infty}$ be an OPS relative to $\sigma$ and $\tau$ a moment functional. Then $\left\langle\tau, P_{n}\right\rangle=0$ for $n>k, k \geq 0$ an integer if and only if $\tau=\phi(x) \sigma$ for some polynomial $\phi(x)$ of degree $\leq k$.

Proof. Assume that $\left\langle\tau, P_{n}\right\rangle=0$ for $n>k$, and consider $\tilde{\tau}=\left(\sum_{j=0}^{k} c_{j} P_{j}\right) \sigma$ where $c_{j}$ are constants. Then $\left\langle\tilde{\tau}, P_{n}\right\rangle=0$ for $n>k$ and so $\tau=\tilde{\tau}$ if and only if $\left\langle\tau, P_{n}\right\rangle=\left\langle\tilde{\tau}, P_{n}\right\rangle$ for $0 \leq n \leq k$. Since $\left\langle\tilde{\tau}, P_{n}\right\rangle=\sum_{j=0}^{k} c_{j}\left\langle\sigma, P_{j} P_{n}\right\rangle=$ $c_{n}\left\langle\sigma, P_{n}^{2}\right\rangle$, we have $\tau=\left(\sum_{j=0}^{k} c_{j} P_{j}\right) \sigma$ with $c_{j}=\left\langle\tau, P_{j}\right\rangle /\left\langle\sigma, P_{j}^{2}\right\rangle, 0 \leq j \leq n$. The converse follows immediately from the orthogonality of $\left\{P_{n}(x)\right\}_{0}^{\infty}$ relative to $\sigma$.

Proof of Theorem 2.1. Let $\left\{P_{n}(x)\right\}_{0}^{\infty}$ be an OPS relative to $\sigma$ with $\left\langle\sigma, P_{m} P_{n}\right\rangle$ $=K_{n} \delta_{m n}, K_{n} \neq 0, m, n=0,1,2, \ldots$. First assume that, for each $n=$ $0,1,2, \ldots, P_{n}(x)$ satisfies equation $(2.5)$. Then we have by using $(2.1),(2.2)$, (2.3), and (2.9)

$$
\begin{aligned}
\lambda_{n} K_{n} \delta_{m n} & =\lambda_{n}\left\langle\sigma, P_{m} P_{n}\right\rangle=\left\langle\sigma, P_{m} L_{4}\left(P_{n}\right)\right\rangle=\left\langle L_{4}\left(P_{n}\right) \sigma, P_{m}\right\rangle \\
& =\left\langle\left[P_{n}^{\prime \prime} \ell_{4} \sigma\right]^{\prime \prime}-\left[P_{n}^{\prime}\left(\frac{1}{2}\left(\ell_{3} \sigma\right)^{\prime}-\ell_{2} \sigma\right)\right]^{\prime}+P_{n} \ell_{0} \sigma, P_{m}\right\rangle \\
& =\left\langle\ell_{4} \sigma, P_{m}^{\prime \prime} P_{n}^{\prime \prime}\right\rangle+\left\langle\frac{1}{2}\left(\ell_{3} \sigma\right)^{\prime}-\ell_{2} \sigma, P_{m}^{\prime} P_{n}^{\prime}\right\rangle+\left\langle\ell_{0} \sigma, P_{m} P_{n}\right\rangle .
\end{aligned}
$$

Hence, we have (2.6) with $\tau_{2}=\ell_{4} \sigma, \tau_{1}=\frac{1}{2}\left(\ell_{3} \sigma\right)^{\prime}-\ell_{2} \sigma, \tau_{0}=\ell_{0} \sigma$, and $M_{n}=\lambda_{n} K_{n}$. To show $\tau_{2} \not \equiv 0$, write $\ell_{4}(x)=\sum_{0}^{4} c_{j} P_{j}(x)$ and assume $\tau_{2} \equiv 0$. Then, for $0 \leq k \leq 4$, we have

$$
0=\left\langle\tau_{2}, P_{k}\right\rangle=\left\langle\ell_{4} \sigma, P_{k}\right\rangle=\sum_{0}^{4} c_{j}\left\langle\sigma, P_{j} P_{k}\right\rangle=c_{k}\left\langle\sigma, P_{k}^{2}\right\rangle .
$$

Hence, we have $c_{k}=0$ for $0 \leq k \leq 4$ and so $\ell_{4}(x) \equiv 0$, which is a contradiction.

Conversely, we assume that $\left\{P_{n}(x)\right\}_{0}^{\infty}$ satisfies (2.6). We may assume that all $P_{n}(x)$ are monic. Consider the equation (2.6) for $m=0,1,2,3,4$.

For $m=0$, we have $\left\langle\tau_{0}, P_{n}\right\rangle=0$ for $n>0$ so that by Lemma 2.4

$$
\tau_{0}=\ell_{0} \sigma
$$

for some polynomial $\ell_{0}(x)$ of degree $\leq 0$.

For $m=1$, we have for $n>1$

$$
0=\left\langle\tau_{1}, P_{n}^{\prime}\right\rangle+\left\langle\tau_{0}, P_{1} P_{n}\right\rangle=-\left\langle\tau_{1}^{\prime}, P_{n}\right\rangle+\left\langle\sigma, \ell_{0} P_{1} P_{n}\right\rangle=-\left\langle\tau_{1}^{\prime}, P_{n}\right\rangle,
$$


so that by Lemma 2.4

$$
\tau_{1}^{\prime}=-\ell_{1} \sigma
$$

for some polynomial $\ell_{1}(x)$ of degree $\leq 1$.

For $m=2$, we have for $n>2$

$$
\begin{aligned}
0 & =\left\langle\tau_{2}, 2 P_{n}^{\prime \prime}\right\rangle+\left\langle\tau_{1}, P_{2}^{\prime} P_{n}^{\prime}\right\rangle+\left\langle\tau_{0}, P_{2} P_{n}\right\rangle \\
& =2\left\langle\tau_{2}^{\prime \prime}, P_{n}\right\rangle-\left\langle\left(P_{2}^{\prime} \tau_{1}\right)^{\prime}, P_{n}\right\rangle+\left\langle\sigma, \ell_{0} P_{2} P_{n}\right\rangle \\
& =2\left\langle\tau_{2}^{\prime \prime}-\tau_{1}, P_{n}\right\rangle+\left\langle\sigma,\left(\ell_{1} P_{2}^{\prime}+\ell_{0} P_{2}\right) P_{n}\right\rangle=2\left\langle\tau_{2}^{\prime \prime}-\tau_{1}, P_{n}\right\rangle,
\end{aligned}
$$

so that by Lemma 2.4

$$
\tau_{2}^{\prime \prime}-\tau_{1}=\ell_{2} \sigma
$$

for some polynomial $\ell_{2}(x)$ of degree $\leq 2$.

For $m=3$, we have for $n>3$

$$
\begin{aligned}
0 & =\left\langle\tau_{2}, P_{3}^{\prime \prime} P_{n}^{\prime \prime}\right\rangle+\left\langle\tau_{1}, P_{3}^{\prime} P_{n}^{\prime}\right\rangle+\left\langle\tau_{0}, P_{3} P_{n}\right\rangle \\
& =12\left\langle\tau_{2}^{\prime}, P_{n}\right\rangle+\left\langle\tau_{2}^{\prime \prime}-\tau_{1}, P_{3}^{\prime \prime} P_{n}\right\rangle+\left\langle\sigma,\left(\ell_{1} P_{3}^{\prime}+\ell_{0} P_{3}\right) P_{n}\right\rangle \\
& =12\left\langle\tau_{2}^{\prime}, P_{n}\right\rangle+\left\langle\sigma,\left(\ell_{2} P_{3}^{\prime \prime}+\ell_{1} P_{3}^{\prime}+\ell_{0} P_{3}\right) P_{n}\right\rangle=12\left\langle\tau_{2}^{\prime}, P_{n}\right\rangle,
\end{aligned}
$$

so that by Lemma 2.4

$$
\tau_{2}^{\prime}=\frac{1}{2} \ell_{3} \sigma
$$

for some polynomial $\ell_{3}(x)$ of degree $\leq 3$.

For $m=4$, we have for $n>4$

$$
\begin{aligned}
0 & =\left\langle\tau_{2}, P_{4}^{\prime \prime} P_{n}^{\prime \prime}\right\rangle+\left\langle\tau_{1}, P_{4}^{\prime} P_{n}^{\prime}\right\rangle+\left\langle\tau_{0}, P_{4} P_{n}\right\rangle \\
& =\left\langle P_{4}^{(4)} \tau_{2}+2 P_{4}^{(3)} \tau_{2}^{\prime}+P_{4}^{\prime \prime} \tau_{2}^{\prime \prime}, P_{n}\right\rangle-\left\langle P_{4}^{\prime \prime} \tau_{1}+P_{4}^{\prime} \tau_{1}^{\prime}, P_{n}\right\rangle+\left\langle P_{4} \tau_{0}, P_{n}\right\rangle \\
& =24\left\langle\tau_{2}, P_{n}\right\rangle+\left\langle\sigma,\left(\ell_{3} P_{4}^{(3)}+\ell_{2} P_{4}^{\prime \prime}+\ell_{1} P_{4}^{\prime}+\ell_{0} P_{4}\right) P_{n}\right\rangle=24\left\langle\tau_{2}, P_{n}\right\rangle,
\end{aligned}
$$

so that by Lemma 2.4

$$
\tau_{2}=\ell_{4} \sigma
$$

for some polynomial $\ell_{4}(x)$ of degree $\leq 4$. With these $\ell_{i}(x)$ thus obtained, we have (2.7) and (2.8), and so (2.9). Moreover, we have from (2.12) and (2.13)

$$
\tau_{1}=\frac{1}{2}\left(\ell_{3} \sigma\right)^{\prime}-\ell_{2} \sigma .
$$

Since $L_{4}\left(P_{n}\right)(x)=\sum_{i=0}^{4} \ell_{i}(x) P_{n}^{(i)}(x)$ is a polynomial of degree $\leq n$, we may write it as $L_{4}\left(P_{n}\right)(x)=\sum_{j=0}^{n} c_{j} P_{j}(x)$ with constants $c_{0}, c_{1}, \ldots, c_{n}$. Then we have for $m=0,1, \ldots, n$ from (2.9), (2.14), and (2.15)

$$
\begin{aligned}
c_{m}\left\langle\sigma, P_{m}^{2}\right\rangle & =\left\langle\sigma, P_{m} \sum_{0}^{n} c_{j} P_{j}\right\rangle=\left\langle\sigma, P_{m} L_{4}\left(P_{n}\right)\right\rangle=\left\langle L_{4}\left(P_{n}\right) \sigma, P_{m}\right\rangle \\
& =\left\langle\left[P_{n}^{\prime \prime} \ell_{4} \sigma\right]^{\prime \prime}-\left[P_{n}^{\prime}\left(\frac{1}{2}\left(\ell_{3} \sigma\right)^{\prime}-\ell_{2} \sigma\right)\right]^{\prime}+P_{n} \ell_{0} \sigma, P_{m}\right\rangle \\
& =\left\langle\tau_{2}, P_{m}^{\prime \prime} P_{n}^{\prime \prime}\right\rangle+\left\langle\tau_{1}, P_{m}^{\prime} P_{n}^{\prime}\right\rangle+\left\langle\tau_{0}, P_{m} P_{n}\right\rangle=M_{n} \delta_{m n} .
\end{aligned}
$$

Hence, we have $c_{m}=0$ for $m=0,1,2, \ldots, n-1$ and so $L_{4}\left(P_{n}\right)=c_{n} P_{n}=$ $\lambda_{n} P_{n}$ by comparing coefficients of $x^{n}$ from both sides. Finally we have $\ell_{4}(x) \not \equiv$ 0 since $\tau_{2}=\ell_{4} \sigma \not \equiv 0$. 
Remark. Note that the term $\ell_{0}(x) y=\ell_{00} y$ may be cancelled from both sides of equation (2.5). In other words, we may take $\ell_{00}$ to be any number in equation (2.5). Therefore, we may require $M_{n} \neq 0, n \geq 0$, since we may have $\lambda_{n} \neq 0$, $n \geq 0$, by taking $\left|\ell_{00}\right|$ to be large.

Inspection of the proof of Theorem 2.1 shows that Theorem 2.1 remains to hold even if we drop the requirements $\ell_{4}(x) \not \equiv 0$ and $\tau_{2} \not \equiv 0$.

Thus, we have as an immediate consequence of Theorem 2.1:

Theorem 2.2. Let $\left\{P_{n}(x)\right\}_{0}^{\infty}$ be an OPS. Then, for each $n=0,1,2, \ldots, P_{n}(x)$ satisfies the second-order differential equation of type

$$
L_{2}(y)=\sum_{i=0}^{2} \ell_{i}(x) y^{(i)}(x)=\sum_{i=0}^{2}\left(\sum_{j=0}^{i} \ell_{i j} x^{j}\right) y^{(i)}(x)=\lambda_{n} y(x)
$$

where $\ell_{2}(x) \not \equiv 0$ and $\lambda_{n}=\ell_{00}+n \ell_{11}+n(n-1) \ell_{22}$ if and only if there are moment functionals $\tau_{1} \not \equiv 0$ and $\tau_{0}$ such that

$$
\left\langle\tau_{1}, P_{m}^{\prime} P_{n}^{\prime}\right\rangle+\left\langle\tau_{0}, P_{m} P_{n}\right\rangle=M_{n} \delta_{m n}, \quad m, n=0,1,2, \ldots,
$$

where $M_{n}$ are constants (which may be taken to be nonzero if one wishes).

Proof. Necessity comes from Theorem 2.1 by taking $\ell_{4}(x) \equiv \ell_{3}(x) \equiv 0$ so that $\tau_{2} \equiv 0, \tau_{1}=-\ell_{2} \sigma$, and $\tau_{0}=\ell_{0} \sigma$. Conversely, equation (2.17) may be understood as equation (2.6) with $\tau_{2} \equiv 0$. Then, each $P_{n}(x)$ must satisfy the differential equation (2.5) with $\ell_{4}(x) \equiv 0$. Then we must have $\ell_{3}(x) \equiv 0$ also since the order of such a differential equation must be even by H. L. Krall's theorem in $\S 1$.

Theorem 2.2 is a restatement of the well-known characterization theorem of classical orthogonal polynomials by Hahn [2]. In fact, it gives a slight improvement of Hahn's theorem as we now show.

Definition 2.2. A polynomial set $\left\{P_{n}(x)\right\}_{0}^{\infty}$ is a weak orthogonal polynomial set (WOPS) if there is a nonzero moment functional $\sigma$ such that

$$
\left\langle\sigma, P_{m} P_{n}\right\rangle=K_{n} \delta_{m n}, \quad m, n=0,1,2, \ldots,
$$

where $K_{n}$ are constants.

The constants $K_{n}$ in Definition 2.2 may be 0 for some $n$ but not for all $n$ since $\sigma \not \equiv 0$.

Theorem 2.3. Let $\left\{P_{n}(x)\right\}_{0}^{\infty}$ be an OPS. Then the following statements are all equivalent.

(i) $\left\{P_{n}(x)\right\}_{0}^{\infty}$ satisfy any one of the two equivalent conditions in Theorem 2.2 .

(ii) $\left\{P_{n+1}^{\prime}(x)\right\}_{0}^{\infty}$ is an OPS.

(iii) $\left\{P_{n+1}^{\prime}(x)\right\}_{0}^{\infty}$ is a WOPS.

Proof. Let $\left\{P_{n}(x)\right\}_{0}^{\infty}$ be an OPS relative to $\sigma$ with $\left\langle\sigma, P_{m} P_{n}\right\rangle=K_{n} \delta_{m n}, K_{n} \neq$ 0 .

(i) $\Rightarrow$ (ii) Assume (i). Then we have

$$
\left\langle-\ell_{2} \sigma, P_{m}^{\prime} P_{n}^{\prime}\right\rangle+\left\langle\ell_{0} \sigma, P_{m} P_{n}\right\rangle=\lambda_{n} K_{n} \delta_{m n}
$$

so that $-\left\langle\ell_{2} \sigma, P_{m}^{\prime} P_{n}^{\prime}\right\rangle=\left[n \ell_{11}+n(n-1) \ell_{22}\right] K_{n} \delta_{m n}$. 
On the other hand, (i) implies that $\left\{P_{n}(x)\right\}_{0}^{\infty}$ must be essentially one of the four classical orthogonal polynomial sets for all of which we have $n \ell_{11}+$ $n(n-1) \ell_{22} \neq 0, n \geq 1$ (see $\left.[1,8]\right)$. Hence, $\left\{P_{n+1}^{\prime}(x)\right\}_{0}^{\infty}$ form an OPS relative to $\ell_{2} \sigma$.

(ii) $\Rightarrow$ (iii) It is trivial from the definition of WOPS.

(iii) $\Rightarrow$ (i) If $\left\{P_{n+1}^{\prime}(x)\right\}_{0}^{\infty}$ is a WOPS relative to $\tau$, then we have (2.17) with $\tau_{1}=\tau$ and $\tau_{0}=\sigma$.

Finally we give the explicit representations of moment functionals $\tau_{2}, \tau_{1}$, and $\tau_{0}$ in Theorem 2.1 for the classical type orthogonal polynomials. We note first that $\tau_{0}$ can be taken to be 0 since we may take $\ell_{0}(x)$ to be 0 in equation (2.5) (cf. (2.10)).

Let $\left\{P_{n}(x)\right\}_{0}^{\infty}$ be an OPS relative to $\sigma$ which satisfies the differential equation (2.5). Then the distributional representation $w(x)$ of $\sigma$ can be obtained by solving equations (2.7) and (2.8) with $w(x)$ instead of $\sigma$ simultaneously in the distribution space (cf. [11]). Then the representations $w_{j}(x)$ of $\tau_{j}, j=1$, 2 , can be obtained immediately from equations (2.14) and (2.15).

In the following examples we follow the notation in [3] and use $H(x)$ to denote the Heaviside step function.

Example 2.1. Legendre type polynomials $L_{n}(x)$ are polynomial solutions of

$$
\left(x^{2}-1\right)^{2} y^{(4)}+8 x\left(x^{2}-1\right) y^{(3)}+(4 \alpha+12)\left(x^{2}-1\right) y^{\prime \prime}+8 \alpha x y^{\prime}=\lambda_{n} y
$$

which are othogonal relative to

$$
w(x)=(\alpha / 2) H\left(1-x^{2}\right)+(1 / 2)[\delta(x-1)+\delta(x+1)] .
$$

Hence, we have from equations (2.14) and (2.15)

$$
w_{1}(x)=\alpha\left[2 \alpha\left(1-x^{2}\right)+4\right] H\left(1-x^{2}\right)
$$

and

$$
w_{2}(x)=(\alpha / 2)\left(x^{2}-1\right)^{2} H\left(1-x^{2}\right)
$$

so that $\left\{L_{n}(x)\right\}_{0}^{\infty}$ has the Sobolev type orthogonality

$$
\begin{aligned}
& (\alpha / 2) \int_{-1}^{1}\left(x^{2}-1\right)^{2} L_{m}^{\prime \prime}(x) L_{n}^{\prime \prime}(x) d x \\
& \quad+\alpha \int_{-1}^{1}\left[2 \alpha\left(1-x^{2}\right)+4\right] L_{m}^{\prime}(x) L_{n}^{\prime}(x) d x=0, \quad m \neq n .
\end{aligned}
$$

Example 2.2. Laguerre type polynomials $R_{n}(x)$ are polynomial solutions of

$$
x^{2} y^{(4)}-\left(2 x^{2}-4 x\right) y^{(3)}+\left[x^{2}-(2 R+6) x\right] y^{\prime \prime}+[(2 R+2) x-2 R] y^{\prime}=\lambda_{n} y
$$

which are othogonal relative to

$$
w(x)=(1 / R) \delta(x)+H(x) \exp (-x) .
$$

Hence, we have from equations (2.14) and (2.15)

$$
w_{1}(x)=2[(R+1) x+1] H(x) \exp (-x)
$$

and 


$$
w_{2}(x)=x^{2} H(x) \exp (-x)
$$

so that $\left\{R_{n}(x)\right\}_{0}^{\infty}$ has the Sobolev type orthogonality

$$
\begin{aligned}
\int_{0}^{\infty} & x^{2} \exp (-x) R_{m}^{\prime \prime}(x) R_{n}^{\prime \prime}(x) d x \\
& +2 \int_{0}^{\infty}[(R+1) x+1] \exp (-x) R_{m}^{\prime}(x) R_{n}^{\prime}(x) d x=0, \quad m \neq n
\end{aligned}
$$

Example 2.3. Jacobi type polynomials $S_{n}(x)$ are polynomial solutions of

$$
\begin{aligned}
& \left(x^{2}-x\right)^{2} y^{(4)}+2 x(x-1)[(\alpha+4) x-2] y^{(3)} \\
& \quad+x\left[\left(\alpha^{2}+9 \alpha+14+2 M\right) x-(6 \alpha+12+2 M)\right] y^{\prime \prime} \\
& \quad+[(\alpha+2)(2 \alpha+2+M) x-2 M] y^{\prime}=\lambda_{n} y \quad(\alpha>-1)
\end{aligned}
$$

which are orthogonal relative to

$$
w(x)=(1 / M) \delta(x)+(1-x)^{\alpha} H\left(x-x^{2}\right) .
$$

Hence, we have from equations (2.14) and (2.15)

$$
w_{1}(x)=2\left[-(\alpha+1+M) x^{2}+(\alpha+M) x+1\right](1-x)^{\alpha} H\left(x-x^{2}\right)
$$

and

$$
w_{2}(x)=x^{2}(1-x)^{2+\alpha} H\left(x-x^{2}\right)
$$

so that $\left\{S_{n}(x)\right\}_{0}^{\infty}$ have the Sobolev type orthogonality

$$
\begin{aligned}
& \int_{0}^{1} x^{2}(1-x)^{2+\alpha} S_{m}^{\prime \prime}(x) S_{n}^{\prime \prime}(\alpha) d x \\
& \quad+2 \int_{0}^{1}\left[-(\alpha+1+M) x^{2}+(\alpha+M) x+1\right](1-x)^{\alpha} S_{m}^{\prime}(x) S_{n}^{\prime}(x) d x \\
& \quad=0, \quad m \neq n .
\end{aligned}
$$

\section{ACKNOWLEDGMENTS}

All of the authors express their sincere thanks to Professor W. N. Everitt who suggested the problem at the 7th Symposium on Orthogonal Polynomials and Applications, Granada, Spain, 1991. The authors thank the referee for kind suggestions which improved the paper; especially, the Sobolev type orthogonality in the last part of the paper.

\section{REFERENCES}

1. S. Bochner, Über Sturm-Liouvillesche Polynomsysteme, Math. Z. 29 (1929), 730-736.

2. W. Hahn, Über die Jacobischen Polynome und zwei verwandte Polynomklassen, Math. Z. 39 (1935), 634-638.

3. A. M. Krall, Orthogonal polynomials satisfying fourth order differential equations, Proc. Roy. Soc. Edinburgh Sect. A 87 (1982), 271-288.

4. A. M. Krall and L. L. Littlejohn, On the classification of differential equations having orthogonal polynomial solutions. II, Ann. Mat. Pura Appl. (4) 4 (1987), 77-102.

5. H. L. Krall, On derivatives of orthogonal polynomials, Bull. Amer. Math. Soc. 42 (1936), 423-428. 
6. __ Certain differential equations for Tchebychev polynomials, Duke Math. J. 4 (1938), 705-719.

7. Colloq. Stud., No. 6, The Pennsylvania State College, State College, PA, 1940.

8. 261-264.

9. K. H. Kwon, S. S. Kim, and S. S. Han, Orthogonalizing weights of Tchebychev sets of polynomials, Bull. London Math. Soc. 24 (1992), 361-367.

10. L. L. Littlejohn, Symmetric factors for differential equations, Amer. Math. Monthly 7 (1983), 462-464.

11. On the classification of differential equations having orthogonal polynomial solutions, Ann. Mat. Pura Appl. (4) 4 (1984), 35-53.

12. L. L. Littlejohn, W. N. Everitt, and S. C. Williams, Orthogonal polynomials in weighted Sobolev spaces, Lecture Notes in Pure and Appl. Math., vol. 117, Marcel Dekker, New York, 1989, pp. 53-72.

13. M. S. Webster, Orthogonal polynomials with orthogonal derivatives, Bull. Amer. Math. Soc. 44 (1938), 880-888.

14. D. V. Widder, The Laplace transform, Princeton Univ. Press, Princeton, NJ, 1972.

(K. H. Kwon, J. K. Lee, and B. H. Yoo) Department of Mathematics, Korea Advanced Institute of Science \& TeChNology, 373-1 Kusong-dong, Yusong-KU, TAejon 305-701, KoREA

E-mail address, K. H. Kwon: khkwon@math1.kaist.ac.kr

(L. L. Littlejohn) Department of Mathematics and Statistics, Utah State University, LOGAN, UTAH 84322-3900

E-mail address: lancelsunf s.math.usu.edu 